\title{
New Limit on T-violating Parameters in Kaon Decays
}

\author{
Marat Khabibullin* \\ Institute for Nuclear Research of Russian Academy of Sciences, pr. 60-letia Oktyabrya, \\ 7a, Moscow 117312, Russia \\ E-mail: 'marat@al20.inr.troitsk.rui
}

M. Abe ${ }^{a}$, M.A. Aliev ${ }^{b}$, V.V. Anisimovsky ${ }^{b}$, M. Aoki ${ }^{c}$, I. Arai ${ }^{a}$, Y. Asano ${ }^{a}$, T. Baker $^{c, d}$, M. Blecher ${ }^{e}$, P. Depommier ${ }^{f}$, M.P. Grigorjev ${ }^{b}$, M. Hasinoff ${ }^{g}$, K. Horie ${ }^{h}$, H.C. Huang ${ }^{i}$, Y. Igarashi ${ }^{c}$, T. Ikeda ${ }^{j}$, J. Imazato $^{c}$, A.P. Ivashkin ${ }^{b}$, A.N. Khotjantsev ${ }^{b}$, Y.G. Kudenko ${ }^{b}$, Y. Kuno ${ }^{c, h}$, L.S. Lee ${ }^{k}$, A.S. Levchenko ${ }^{b}$, G.Y. Lim ${ }^{k, c}$, J.A. Macdonald ${ }^{l}$, D.R. Marlow ${ }^{m}$, C.R. Mindas ${ }^{m}$, O.V. Mineev ${ }^{b}$, C. Rangacharyulu ${ }^{d}$, S. Shimizu ${ }^{h}$, Y.-M. Shin ${ }^{d}$, A. Suzuki ${ }^{a}$, A. Watanabe ${ }^{a}$, N.V. Yershov ${ }^{b}$, T. Yokoi ${ }^{c} \dagger$ (E246 KEK-PS COLLABORATION)

\begin{abstract}
A search for a T-violating transverse muon polarization $\left(\mathrm{P}_{T}\right)$ in $K^{+} \rightarrow \pi^{0} \mu^{+} \nu$ decay was carried out in the experiment E246 at the KEK-PS. The Standard model (SM) predicts vanishing value of the polarization $\left(<10^{-7}\right)$, so an observation of non-zero $\mathrm{P}_{T}$ at the current experimental sensitivity level would be a definite signature of new physics beyond SM. The preliminary result of the analysis of the 1996-1998 data is presented here: $\mathrm{P}_{T}=(-3.3 \pm 3.7$ (stat $) \pm 0.9($ syst $\left.)\right) \times 10^{-3}$, which corresponds to the $\mathrm{T}$-violation parameter $\operatorname{Im}(\xi)=(-0.9 \pm 1.2($ stat $) \pm 0.3($ syst $)) \times 10^{-2}$. There is no evidence for T-violation in $K_{\mu 3}$ at the current level of sensitivity.
\end{abstract}

\section{Introduction}

This talk is about a search for T-violation in the decay $K^{+} \rightarrow \pi^{0} \mu^{+} \nu\left(K_{\mu 3}^{+}\right)$. The Lorentz invariant matrix element of this decay can be written as

$$
M=\left(G_{F} / 2\right) \sin \theta_{C}\left[f_{+}\left(q^{2}\right)\left(p_{K}+p_{\pi}\right)^{\lambda}+f_{-}\left(q^{2}\right)\left(p_{K}-p_{\pi}\right)^{\lambda}\right]\left[\bar{u}_{\mu} \gamma_{\lambda}\left(1-\gamma_{5}\right) u_{\nu}\right]
$$

\section{${ }^{*}$ Speaker.}

${ }^{\dagger a}$ University of Tsukuba, 305-0006, Japan; ${ }^{b}$ Institute for Nuclear Research of RAS, Moscow 117312, Russia; ${ }^{c}$ KEK, 305-0801, Japan; ${ }^{d}$ University of Saskatchewan, S7N 0W0, Canada; ${ }^{e}$ Virginia Polytechnic Institute and State University, USA; ${ }^{f}$ University of Montreal, H3C 3J7, Canada; ${ }^{g}$ University of British Columbia, Vancouver, V6T 1 Z1 Canada; ${ }^{h}$ Osaka University, 560-0043, Japan; ${ }^{i}$ National Taiwan University, Taipei, Taiwan; ${ }^{j}$ Institute of Physical and Chemical Research, Hirosawa 2-1, Japan; ${ }^{k}$ Korea University, Seoul 136-701, Korea; ${ }^{l}$ TRIUMF, V6T 2A3, Canada; ${ }^{m}$ Princeton University, NJ 08544, USA. 
where $G_{F}$ is the Fermi constant, $\theta_{C}$ - the Cabibbo angle, $p_{K}$ and $p_{\pi}$ are 4-momenta of the kaon and pion, respectively, and $q$ is the 4-momentum transfer to leptons. It includes two hadronic form factors ${ }^{1} f_{-}$and $f_{+}$which should be relatively real in the case of T-invariance: $\operatorname{Im}(\xi)=0$, where $\xi \equiv f_{-}\left(q^{2}\right) / f_{+}\left(q^{2}\right)\left[i_{1}^{1}\right]$.

On the other hand, the only observable related to T-violation in $K_{\mu 3}^{+}$decay is a transverse muon polarization $\mathrm{P}_{T}$ defined as a muon polarization component normal to the decay plane: $\mathrm{P}_{T}=\vec{s}_{\mu} \cdot\left(\vec{p}_{\pi^{0}} \times \vec{p}_{\mu^{+}}\right) /\left|\vec{p}_{\pi^{0}} \times \vec{p}_{\mu^{+}}\right|$, where $\vec{s}_{\mu}$ is the muon spin, $\vec{p}_{\pi^{0}}$ and $\vec{p}_{\mu^{+}}$are the $\pi^{0}$ and $\mu^{+}$momentum vectors, respectively. It's known that $\mathrm{P}_{T}$ is proportional to $\operatorname{Im}(\xi)$ : $\mathrm{P}_{\mathrm{T}} \sim m_{\mu} \cdot m_{K} \cdot \operatorname{Im}(\xi)$, where $m_{\mu}$ and $m_{K}$ are muon and kaon masses, so by measuring the transverse polarization, one gets an information on T-violation.

What level of $\mathrm{P}_{\mathrm{T}}$ should be expected from a theoretical point of view? The Standard Model (SM) predicts zero, or $<10^{-7}$ [i [i] $]$, which is far below the current experimental sensitivity. A contribution of the final state interactions (FSI) was found to be below the $10^{-5}$ level [5] , so this decay presents a good opportunity to search for new physics beyond SM. Indeed, some extensions to the SM, such as multi Higgs doublet models, supersymmetrical models with/without R-parity violation (SUSY), and leptoquark models predict $\mathrm{P}_{T}<10^{-2}$ [i6]"

The previous experiment carried out at BNL almost 20 years ago found no evidence for T-violation: $\mathrm{P}_{T}=(-3.1 \pm 5.3) \times 10^{-3}$, which corresponds to $\operatorname{Im}(\xi)=(-1.6 \pm 2.5) \times 10^{-2}[\underline{i}]$. The latest result on transverse polarization was published by our group two years ago (19961997 data $): \mathrm{P}_{T}=(-4.2 \pm 4.9($ stat $) \pm 0.9($ syst $)) \times 10^{-3}$ and $\operatorname{Im}(\xi)=(-1.3 \pm 1.6($ stat $) \pm$

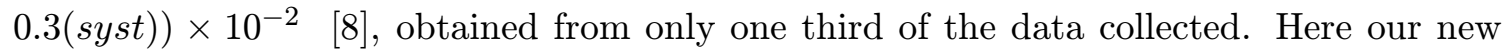
result based on increased statistics including the 1998 data set is presented.

\section{Experimental Technique}

The main feature of our experiment is the use of stopped kaons which allows detection of decay particles from a wide kinematic range. Kaons with $660 \mathrm{MeV} / \mathrm{c}$ momentum are identified by a Čerenkov detector, degraded and stopped in an active target made of 256 scintillating fibers (Fig İ, a). A decay muon passes through the target, ring hodoscope, magnetic spectrometer with three proportional chambers $(\mathrm{C} 2, \mathrm{C} 3, \mathrm{C} 4)$ and enters a muon polarimeter consisting of a copper degrader, pure aluminium stopper and positron counters. The neutral pion almost instantly decays into two photons which are detected by a $\mathrm{CsI}(\mathrm{Tl})$ calorimeter comprised of 768 crystal modules $[\underline{\overline{9}}]$.

The experimental setup consists of 12 azimuthally symmetric sectors, which correspond to the gaps in the superconducting toroidal magnet (Fig $\underline{1}_{1}^{\overline{1}}$ b). Each of 12 polarimeters has two positron counters detecting $e^{+} \mathrm{s}$ from the muon decays in clockwise $(c w)$ and counterclockwise (ccw) directions (Fig. $\left.\overline{2}_{1}^{\prime}\right)$. The difference in the number of hits between these two counters is an asymmetry representing the transverse polarization $\mathrm{P}_{T}$ of interest.

\footnotetext{
${ }^{1}$ The matrix element $\left({ }_{1}^{-1} \cdot \overline{1}_{1}^{1}\right)$ doesn't include explicitly any exotic terms, since our recent study of $K^{+} \rightarrow$ $\pi^{0} e^{+} \nu\left(K_{e 3}^{+}\right)$decays shows that, first, scalar $f_{S}$ and tensor $f_{T}$ form factors are consistent with zero [2] and, second, there is no deviation from $\mu$-e universality
} 


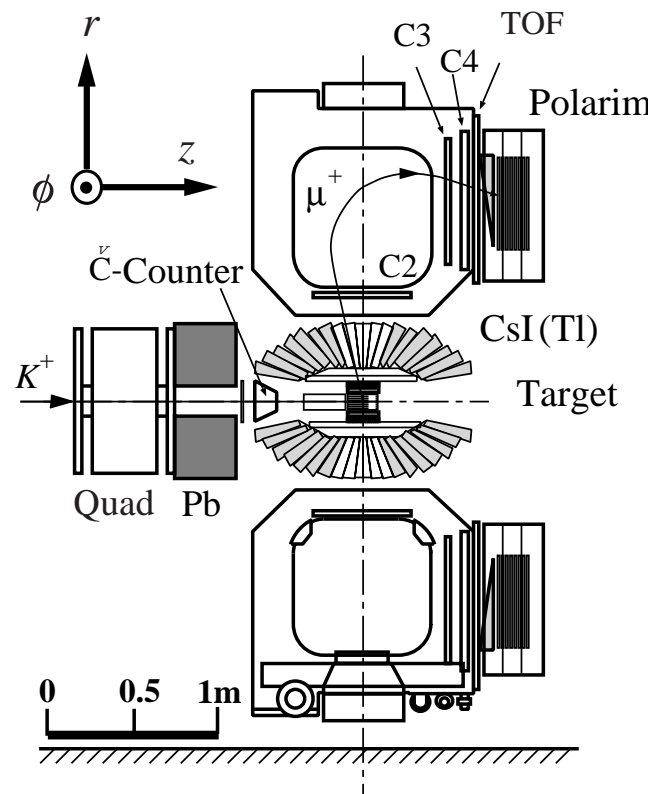

(a)

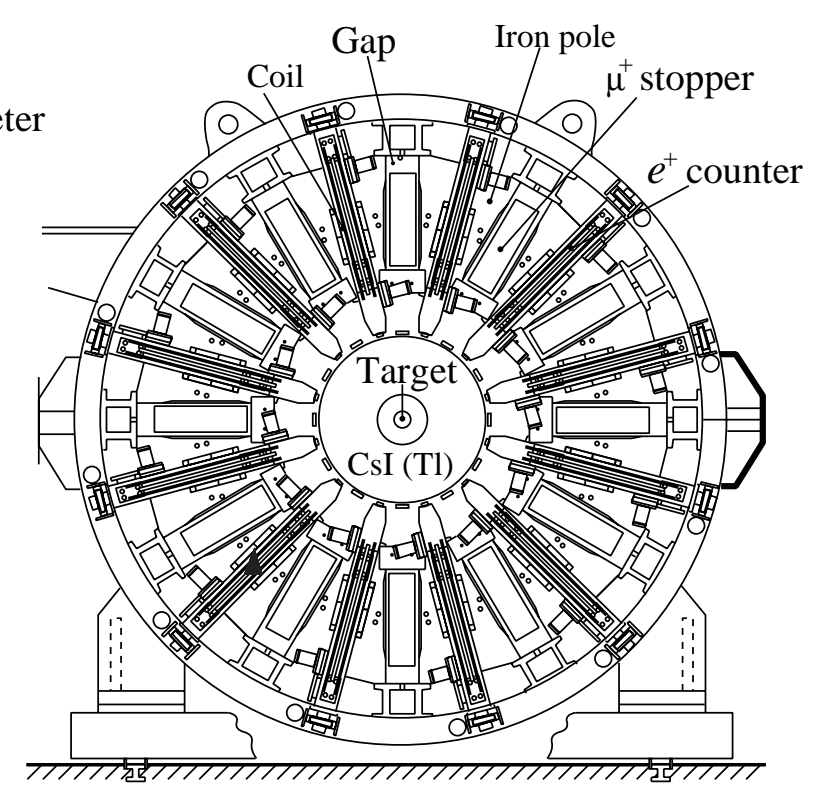

(b)

Figure 1: E246 experimental setup; (a) cross section side view, (b) end view.

With decays of stopped kaons events are divided into two classes: with backward$(b w d)$, and forward- $(f w d)$ going pions (with respect to the initial kaon beam direction). Since events with opposite $\pi^{0}$ directions have opposite signs of $\mathrm{P}_{T}$, combining of the events with $f w d$ - and bwd-going pions increases the sensitivity to $\mathrm{P}_{T}$ ("double ratio"). It also reduces the effect of various geometrical factors whose contribution to $\mathrm{P}_{T}$ is independent of the $\pi^{0}$ direction ("fwd/bwd cancellation").

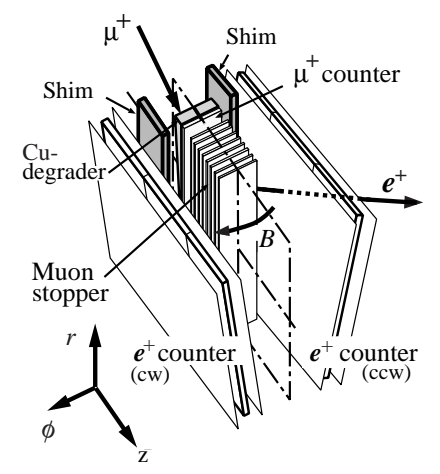

Figure 2: Muon polarimeter of each sector consists of copper degrader, aluminium stopper and two plastic positron counters (clockwise and counter-clockwise with respect to the muon).

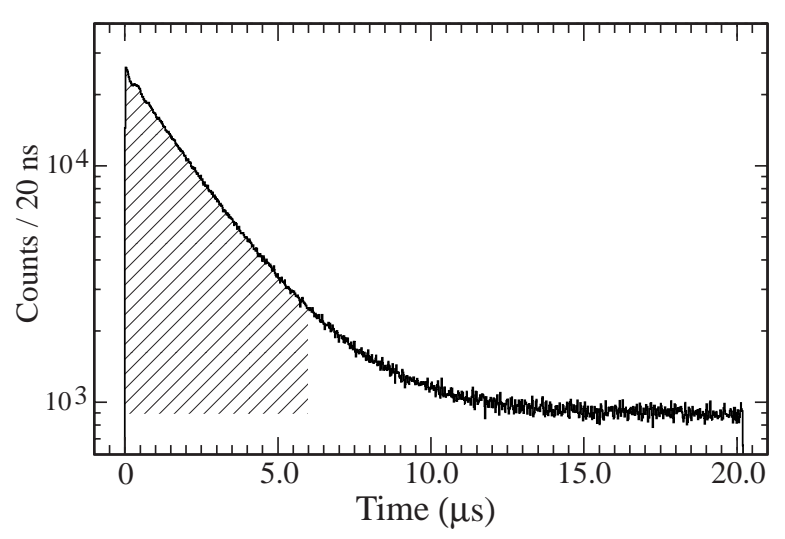

Figure 3: Positron time spectrum of all good events (sum of $2 \gamma$ and $1 \gamma$ ). The hatched region is the analyzed signal region after subtraction of the constant background. 
In order to minimize the background level a number of kinematic cuts were applied. The $2.6 \mathrm{MeV} / \mathrm{c}$ momentum resolution $(\sigma$ at $205 \mathrm{MeV} / \mathrm{c}$ ) of the spectrometer allows the momentum cut to be set as high as possible and reject most of $K^{+} \rightarrow \pi^{+} \pi^{0}$ events. $K^{+} \rightarrow e^{+} \pi^{0} \nu$ events are eliminated by means of time-of-flight. Neutral pions from the $K_{\mu 3}$ are identified either by two photons in $\mathrm{CsI}(\mathrm{Tl})$ by means of the invariant mass $M_{\gamma \gamma}$ cut $75 \mathrm{MeV} / \mathrm{c}^{2}<M_{\gamma \gamma}<160 \mathrm{MeV} / \mathrm{c}^{2}$ (" $2 \gamma$-events"), or by one detected photon with an energy $E_{\gamma}>70 \mathrm{MeV}$ when the second photon has escaped detection ("1 1 -events"). A selection of events with $f w d(b w d)$-going $\pi^{0} \mathrm{~s}$ is achieved by applying the proper polar angle cut: $\theta_{\pi^{0}}<70^{\circ}$ for fwd and $\theta_{\pi^{0}}>110^{\circ}$ for $b w d$, where $\theta_{\pi^{0}}$ is the angle between the $\pi^{0}$ momentum and the kaon beam axis (Z-axis).

\section{Systematics and Results}

After selecting $K_{\mu 3}$ events the number of hits $N_{c w}\left(N_{c c w}\right)$ in the signal regions (from $20 \mathrm{~ns}$ to $6 \mu \mathrm{s}$ ) of the positron counter time spectra is determined ( Fig. $\overline{3}$ in). The T-violation asymmetry $A_{T}$ is then extracted as

$$
\mathrm{A}_{T}=\frac{1}{4}\left[\frac{\left(N_{c w} / N_{c c w}\right)_{f w d}}{\left(N_{c w} / N_{c c w}\right)_{b w d}}-1\right]
$$

Then, the transverse polarization $\mathrm{P}_{T}$ is obtained as $\mathrm{P}_{T}=\mathrm{A}_{T} /\left(\alpha\left\langle\cos \theta_{T}\right\rangle\right)$, where the geometrical attenuation factor $\left\langle\cos \theta_{T}\right\rangle$ is calculated by Monte Carlo simulations, and the analyzing power $\alpha=0.197 \pm 0.005$ is extracted using events with transverse-going pi-

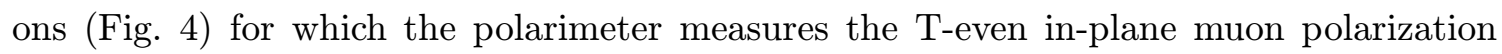

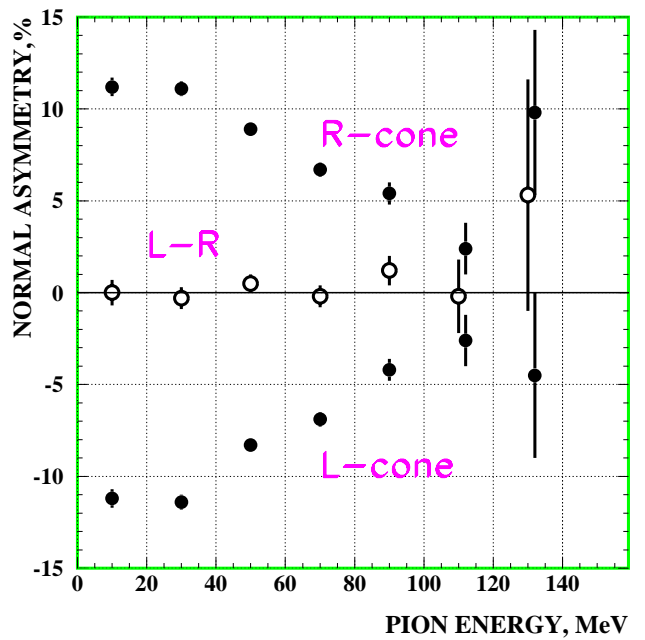

Figure 4: Normal asymmetry $A_{N}$ vs $\pi^{0}$ energy. In this case the kaon decay plane is perpendicular to Z-axis, $\pi^{0}$ goes either in the right $(\mathrm{R})$ or left $(\mathrm{L})$ cone with respect to the muon direction. The events with opposite $\pi^{0}$ directions have opposite signs of $A_{N}$.

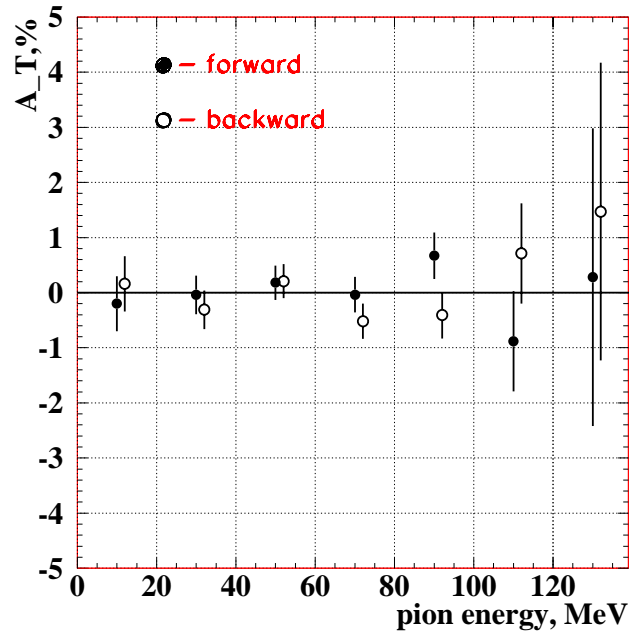

Figure 5: Transverse asymmetry $A_{T}$ vs $\pi^{0}$ energy. Empty circles present the events with backward going $\pi^{0}$; black circles - "forward" events. 


\begin{tabular}{|c|c|}
\hline Source of error & $\mathbf{\Delta P}_{\mathbf{T}} \times \mathbf{1 0}^{-\mathbf{4}}$ \\
\hline \hline CsI(Tl) misalignment & 1.6 \\
\hline Decay plane rotation & 2.2 \\
\hline Misalignment of positron counters & 2.3 \\
\hline MWPC $\phi$-offset & 2.5 \\
\hline$K^{+}$stopping distribution & $<3.0$ \\
\hline Analysis & 3.8 \\
\hline \hline Fringing field asymmetry & 6.1 \\
\hline Total & $\mathbf{9 . 2}$ \\
\hline
\end{tabular}

Table 1: Main systematic errors

$P_{N}\left(\perp p_{\mu}\right)$. T-violation parameter $\operatorname{Im}(\xi)$ is calculated then as $\operatorname{Im}(\xi)=\mathrm{P}_{T} / \Phi$, where $\Phi \approx 0.3$ is a kinematic factor obtained from the analysis of the $K_{\mu 3}$ Dalitz-plot. The 12 -fold rotational symmetry and fwd/bwd cancellation allow most of the systematic errors to be suppressed by a factor of 20-30, so that the resulting uncertainty of $\mathrm{P}_{T}$ due to the system-

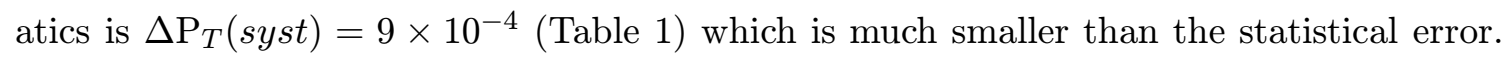
A check of the instrumental systematics is demonstrated in Fig. ${ }_{1}^{15}$ where the dependence of the asymmetry $\mathrm{A}_{T}$ on the $\pi^{0}$-energy is plotted. As in the case of the normal asymmetry $\mathrm{A}_{N}$ (Fig. $\overline{i_{i}}$ ) there is no visible systematic shift for $\mathrm{A}_{T}$.

Two thirds of all the data collected during 1996-2000 runs have been analyzed by this time $\left(\sim 7.2 \times 10^{6} K_{\mu 3}\right.$ events). Our preliminary result shows no T-violation in $K_{\mu 3}^{+}$at the current experimental sensitivity: $\mathrm{P}_{T}=(-3.3 \pm 3.7$ (stat) \pm 0.9 (syst) $) \times 10^{-3}$ and $\operatorname{Im}(\xi)=(-0.9 \pm 1.2($ stat $) \pm 0.3($ syst $)) \times 10^{-2}$.

\section{References}

[1] N. Cabibbo and A. Maksymowicz, 'Phys. Lett. $\mathbf{9}(1964)$ 352;; - $360(\mathrm{E}) ;$;

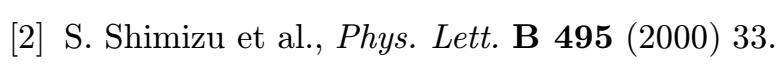

[3] K. Horie et al., Phys. Lett. B $\mathbf{5} 13 \mathbf{3}(2001) 311$.

[4] E. Golowich and G. Valencia, $P$ hyss. Rev. $\mathbf{D} \mathbf{4} 0(1989) 1 \overline{1} \overline{2}_{r}^{\prime}$

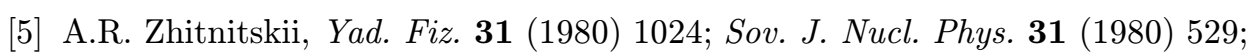

V.P. Efrosinin et al., Phys. Lett. B $\mathbf{4} 93$ 3 $(2000) 293$

[6] C.Q. Geng and S.K. Lee, Phys. Rev. D 51 (1995) 99;;

G.-H. Wu and J.N. Ng, 'Phys.Lett. B 392 (1997) 93

M. Fabbrichesi and F. Vissani, $P$ hys. Rev. D $\mathbf{5} 5$ (1997) 5334

G. Bélanger and C.Q. Geng, 'Phys.Rev. D 44 (1991) 2789.

[7] S.R. Blatt et al., 'P

[8] M. Abe et al., PPhys. Rev. Lett. 83 (1999) $42 \overline{2}$ 3.

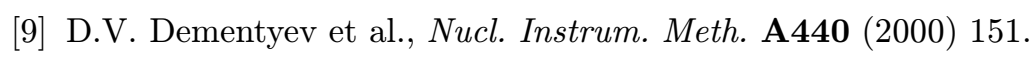

